\title{
Article \\ Extended Fuzzy Sets and Their Applications
}

\author{
Bahram Farhadinia $1, *$ (D) and Francisco Chiclana $2,3, *$ (D) \\ 1 Department of Mathematics, Quchan University of Technology, Razavi Khorasan Province, \\ Quchan 9477177870, Iran \\ 2 Institute of Artificial Intelligence, De Montfort University, Leicester LE1 9BH, UK \\ 3 Andalusian Research Institute on Data Science and Computational Intelligence (DaSCI), University of \\ Granada, 18071 Granada, Spain \\ * Correspondence: bfarhadinia@qiet.ac.ir (B.F.); chiclana@dmu.ac.uk (F.C.)
}

Citation: Farhadinia, B.; Chiclana, F. Extended Fuzzy Sets and Their Applications. Mathematics 2021, 9 , 770. https://doi.org/10.3390/ math 9070770

Received: 15 March 2021

Accepted: 30 March 2021

Published: 2 April 2021

Publisher's Note: MDPI stays neutral with regard to jurisdictional claims in published maps and institutional affiliations.

Copyright: (c) 2021 by the authors. Licensee MDPI, Basel, Switzerland. This article is an open access article distributed under the terms and conditions of the Creative Commons Attribution (CC BY) license (https:/ / creativecommons.org/licenses/by/ $4.0 /)$.

\begin{abstract}
This contribution deals with introducing the innovative concept of extended fuzzy set (E-FS), in which the S-norm function of membership and non-membership grades is less than or equal to one. The proposed concept not only encompasses the concept of the fuzzy set (FS), but it also includes the concepts of the intuitionistic fuzzy set (IFS), the Pythagorean fuzzy set (PFS) and the p-rung orthopair fuzzy set (p-ROFS). In order to explore the features of the E-FS concept, set and algebraic operations on E-FSs, average and geometric operations of E-FSs are studied and an E-FS score function is defined. The superiority of the E-FS concept is further confirmed with a score-based decision making technique in which the concepts of FS, IFS, PFS and p-ROFS do not make sense.
\end{abstract}

Keywords: fuzzy set (FS); intuitionistic fuzzy set (IFS); pythagorean fuzzy set (PFS); p-rung orthopair fuzzy set (p-ROFS); extended fuzzy set (E-FS); decision making

\section{Introduction}

Decision making is one of the most important and critical activities of the human being. However, human beings' opinions or preferences are pervaded with vagueness and imprecision. Zadeh [1] proposed a new methodology to address vagueness and imprecision based on the concept of 'fuzziness or gradual degree of membership' to a set, which was term 'fuzzy set' (FS). Since then, fuzzy sets have been extensively studied and extended with other types of fuzziness based sets: intuitionistic fuzzy set (IFS) [2], Pythagorean fuzzy set (PFS) [3] and p-rung orthopair fuzzy set (p-ROFS) [4] . The definitions of FS, IFS, PFS and p-ROFS rely on the concepts of membership and non-membership degrees of an element to a set. In the case of FSs, the sum of membership and non-membership degrees of an element to a set is constrained to be equal to one; for IFSs, this sum is constrained to be less than or equal to one; while constraints on the sum of powers of membership and non-membership degrees to be less than or equal to one are used in the case of PFS (powers of two) and p-ROFSs ( $p$-power). Recall that if $p=1$ and $p=2$, then a p-ROFS reduces to an IFS and a PFS, respectively, making IFS and PFS special cases of the p-ROFS.

The concepts of FS, IFS, PFS and p-ROFS have been applied to a wide-range of decision making problems including aggregation-based studies, information measure-based research and ranking-based developments [5-8]. Among a large number of aggregationbased studies, we highlight those carried out by $\mathrm{Xu}$ and Yager [9], where geometric aggregation operators were introduced, and $\mathrm{He}$ et al. [10] where the geometric interaction average operators for IFSs was proposed. By using Einstein operations, Garg [11] presented the concept of information and geometric aggregations for PFSs; Liu and Wang [12] extended the weighted average and geometric operators to p-ROFSs, while Wei et al. [13] and Peng et al. [14] investigated the Heronian mean and an exponential operations for p-ROFSs, respectively. Abundant information measure-based research studies have been dedicated to the concepts of FS, IFS, PFS and p-ROFS. Wu et al. [15] exploited the isomorphism between IFSs and interval-valued FSs to formally develop an approach to consistency of IFSs. 
Garg and Kumar [16] studied a class of similarity measures of IFSs, while Wu and Chiclana in [17] and Ureña et al. in [18] applied IFSs approaches to estimate missing preferences and consensus building. Zhang [19] worked on a multiple criteria group decision making technique of PFSs, and Guolin et al. [20] proposed a new decision-theoretic rough set model with p-ROFSs.

An important topic worth attention is how to compare and rank FSs, IFSs, PFSs or p-ROFSs. Xu and Yager [9] presented a score and accuracy functions on IFSs. Peng [21] introduced a score function for PFSs and then investigated a number of its properties. Farhadinia and Liao [22] first reviewed the existing score functions for p-ROFSs, and then proposed a parametrised score function of $\mathrm{p}$-ROFSs.

It is worth noting that, as $p$ value increases in p-ROFSs, the space of acceptable orthopairs increases and more orthopairs satisfy the boundary constraint. Therefore, we can express a wider range of fuzzy information by using p-ROFSs than using FSs, IFSs or PFSs. However, it is observed that their approaches are limited in nature. For example, if an expert provides the value 0.95 for membership degree and the value 0.9 for nonmembership degree, then $0.95+0.9 \neq 1,0.95+0.9 \not \leq 1,(0.95)^{2}+(0.9)^{2} \not \leq 1$, while $(0.95)^{p}+(0.9)^{p} \leq 1$ when $p \geq 10$, which restricts the option of selecting $p$ in p-ROFS theory. Hence, FSs, IFSs, PFSs and p-ROFSs cannot describe this information properly and effectively. To manage such a situation, and releasing the restriction of $p$ selection in p-ROFS theory, the concept of extended fuzzy set (E-FS) is introduced herein with a S-norm function of the membership and non-membership degrees being constrained to be less than or equal to 1 . The proposed E-FS concept is therefore more effective and more general than the existing concepts for handling uncertain information in real-life decision processes. Indeed, our proposed E-FS concept expresses a wider range of fuzzy information than p-ROFS without needing to consider the extra parameter $p$, which is essential in defining a p-ROFS. In addition, the proposed E-FS diminishes the restriction that FS, IFS, PFS and p-ROFS impose on membership grades and it provides decision-makers with more elasticity to express their opinions according to the membership grades of an element than the concepts of FS, IFS, PFS and p-ROFS.

Summarising, this study main research contributions are:

1. The introduction of the concept of E-FS, as an overarching concept of FS to include the concepts of IFS, PFS and p-ROFS;

2. The study of the fundamental principles of E-FSs in comparison to FSs, IFSs, PFSs and p-ROFSs;

3. The definition of some algebraic and set operations on E-FSs, including the average and geometric operations on E-FSs;

4. The presentation of a score function for E-FSs.

The rest of this contribution is organised as follows-Section 2 provides a brief review of some preliminaries needed before the concept of E-FS is introduced. Sections 3-5 are devoted to the study of set and algebraic operations on E-FSs, average and geometric operations of E-FSs and a score function of E-FSs, respectively. Section 6 presents the application of the proposed E-FS score function to solve a decision making problem. Conclusions are drawn in Section 7.

\section{Fundamental Principles}

This section is devoted to reviewing some existing basic concepts and notions that are of importance in fuzzy set theory. Then, we deal with the main part of this contribution, which is the introduction of the concept of extended fuzzy set (E-FS).

Formally, given a universal set $X$, a set $A$ on $X$ is characterised by two functions on $X$ that measure the degree of membership $\left(\mu_{A}(x)\right)$ and the degree of non-membership $\left(v_{A}(x)\right)$ to $A$ of each element of the universal set $x \in X$.

- $\quad A$ is a classical set (CS) when $\mu_{A}$ and $v_{A}$ range is $\{0,1\}$ and verify the property

$$
\mu_{A}(x)+v_{A}(x)=1, \quad \forall x \in X .
$$


In this case, the membership function is known as a characteristic function and usually denoted by $\delta_{A}$; the non-membership function is uniquely defined by (1).

- $\quad A$ is a fuzzy set (FS) when $\mu_{A}$ and $v_{A}$ range is $[0,1]$ and verify the property

$$
\mu_{A}(x)+v_{A}(x)=1, \quad \forall x \in X
$$

Consequently, as with CSs, the non-membership function $v_{A}$ is uniquely defined from the membership function.

- $A$ is an intuitionistic fuzzy set (IFS) when $\mu_{A}$ and $v_{A}$ range is $[0,1]$ and verify the property

$$
\mu_{A}(x)+v_{A}(x) \leq 1, \quad \forall x \in X .
$$

In this case, given a membership function, multiple non-membership functions verifying (3) may exist. The concept of hesitancy is therefore present in an IFS, which is modelled in this framework via the hesitancy function $\pi_{A}=1-\left(\mu_{A}(x)+v_{A}(x)\right)$. For IFSs, membership and non-membership functions are specifically denoted as $\mu_{A_{I F S}}$ and $v_{A_{I F S}}$, respectively.

- $A$ is a Pythagorean fuzzy set (PFS) when $\mu_{A}$ and $v_{A}$ range is $[0,1]$ and verify the property

$$
\mu_{A}^{2}(x)+v_{A}^{2}(x) \leq 1, \quad \forall x \in X .
$$

As with IFSs, a membership function may have associated more than one nonmembership functions as per (4). For PFSs, membership and non-membership functions are specifically denoted as $\mu_{A_{P F S}}$ and $v_{A_{P F S}}$, respectively.

- $\quad A$ is a p-rung orthopair fuzzy set (p-ROFS) $(p \geq 1)$ when $\mu_{A}$ and $v_{A}$ range is $[0,1]$ and verify the property

$$
\mu_{A}^{p}(x)+v_{A}^{p}(x) \leq 1, \quad \forall x \in X .
$$

If $p=2$, then (5) becomes (4); while if $p=1$, then (5) becomes (3).

It is obvious from examining the above set concept definitions that all of them follow the same pattern in that all impose a constraint to the membership and non-membership functions of the type

$$
\left\{\begin{array}{l}
\phi:[0,1] \times[0,1] \longrightarrow[0,1] \\
\phi\left(\mu_{A}(x), v_{A}(x)\right) \in[0,1], \quad \forall x \in X .
\end{array}\right.
$$

A CS requires that $\mu_{A}(x), v_{A}(x) \in\{0,1\}$, and can be seen as a subclass of FS, IFS, PFS or p-ROFS. Although, the concept of p-ROFS has been widely studied by researchers, very little is known about the pre-determination of the parameter $p$. This motivates us to propose the concept of extended fuzzy set (E-FS), which is not dependent on any value of $p$.

Definition 1. Consider the referential set $X$. An extended fuzzy set (E-FS) $A_{E-F S}$ on $X$ is characterised by two functions, $\mu_{A_{E-F S}}: X \rightarrow[0,1]$ and $v_{A_{E-F S}}: X \rightarrow[0,1]$, called the membership and non-membership functions of $A_{E-F S}$, respectively, that verify the property

$$
0 \leq \mu_{A_{E-F S}}(x) \odot v_{A_{E-F S}}(x) \leq 1, \quad \forall x \in X,
$$

where $\odot$ is a S-norm or union function. Using set theoretic notation, an extended fuzzy set (E-FS) on $X$ will be denoted as follows

$$
A_{E-F S}=\left\{\left\langle x, \mu_{A_{E-F S}}(x), v_{A_{E-F S}}(x)\right\rangle: x \in X\right\} .
$$

Recall that an S-norm is a binary function $\odot:[0,1] \times[0,1] \rightarrow[0,1]$ that satisfies the following properties (see e.g., [23])

1. $\odot(x, 0)=x$ (boundary condition);

2. $\forall x, y, z \in[0,1]$, if $y \leq z$, then $\odot(x, y) \leq \odot(x, z)$ (monotonicity); 
3. $\forall x, y \in[0,1], \odot(x, y)=\odot(y, x)$ (commutativity);

4. $\forall x, y, z \in[0,1], \odot(x, \odot(y, z))=\odot(\odot(x, y), z)$ (associativity).

The following S-norms are respectively known as Algebraic, Einstein, Hamacher and Frank norms:

$$
\begin{aligned}
& \odot_{1}(x, y)=x+y-x y ; \\
& \odot_{2}(x, y)=\frac{x+y}{1+x y} ; \\
& \odot_{3}^{\epsilon}(x, y)=\frac{x+y-x y-(1-\epsilon) x y}{1-(1-\epsilon) x y}, \quad \epsilon>0 ; \\
& \odot_{4}^{\epsilon}(x, y)=1-\log _{\epsilon}\left(1+\frac{\left(\epsilon^{1-x}-1\right)\left(\epsilon^{1-y}-1\right)}{\epsilon-1}\right), \quad \epsilon>1 .
\end{aligned}
$$

Proposition 1. Any FS, IFS, PFS and $p$-ROFS on $X$ is an E-FS on X.

Proof of Proposition 1 is in Appendix A.1.

Remark 1. The converse is not true. Indeed, an E-FS may not necessarily be a p-ROFS for all values of $p \in[1, \infty)$. For instance, the $E-F S\left\{\left(\mu_{A_{E-F S}}(x), v_{A_{E-F S}}(x)\right)\right\}=\{(0.95,0.9)\}$ is not a $p$-ROFS for any $p \in[1,10)$.

Remark 2. In order to simplify the following discussions, $\left(\mu_{A_{E-F S}}(x), v_{A_{E-F S}}(x)\right)$ is called an extended fuzzy number (E-FN). This is nothing else than a special case of E-FS. Furthermore, since the same treatment strategy will be applied for all types of S-norm $\odot$, we only consider $\odot:=\odot_{1}$ in what follows.

\section{Set and Algebraic Operations on E-FNs}

The usual operations of addition and multiplication between E-FSs are denoted by $\oplus$ and $\otimes$ while the operation relating the membership and non-membership degrees of an E-FS is denoted by $\odot_{1}$. We now propose a number of set and algebraic operations on E-FNs.

Definition 2. For any E-FNs $A_{E-F N}=\left(\mu_{A_{E-F N}}, v_{A_{E-F N}}\right)$ and $B_{E-F N}=\left(\mu_{B_{E-F N}}, v_{B_{E-F N}}\right)$, the following operations are defined:

$$
\begin{aligned}
& A_{E-F N}^{c}=\left(\mu_{A_{E-F N}^{c},}, v_{A_{E-F N}^{c}}\right)=\left(v_{A_{E-F N}}, \mu_{A_{E-F N}}\right) ; \\
& A_{E-F N} \cap B_{E-F N}=\left(\mu_{A_{E-F N} \cap B_{E-F N}}, v_{A_{E-F N} \cup B_{E-F N}}\right) \\
& =\left(\min \left\{\mu_{A_{E-F N}}, \mu_{B_{E-F N}}\right\}, \max \left\{v_{A_{E-F N}}, v_{B_{E-F N}}\right\}\right) ; \\
& A_{E-F N} \cup B_{E-F N}=\left(\mu_{A_{E-F N} \cup B_{E-F N}}, v_{A_{E-F N} \cap B_{E-F N}}\right) \\
& =\left(\max \left\{\mu_{A_{E-F N}}, \mu_{B_{E-F N}}\right\}, \min \left\{v_{A_{E-F N}}, v_{B_{E-F N}}\right\}\right) ; \\
& A_{E-F N} \oplus B_{E-F N}=\left(\mu_{A_{E-F N} \oplus B_{E-F N}}, v_{A_{E-F N} \oplus B_{E-F N}}\right) \\
& =\left(1-\left(1-\mu_{A_{E-F N}}\right)\left(1-\mu_{B_{E-F N}}\right), v_{A_{E-F N}} v_{B_{E-F N}}\right) ; \\
& A_{E-F N} \otimes B_{E-F N}=\left(\mu_{A_{E-F N} \otimes B_{E-F N}}, v_{A_{E-F N} \otimes B_{E-F N}}\right) \\
& =\left(\mu_{A_{E-F N}} \mu_{B_{E-F N}}, 1-\left(1-v_{A_{E-F N}}\right)\left(1-v_{B_{E-F N}}\right)\right) \text {; } \\
& \lambda A_{E-F N}=\left(\mu_{\lambda A_{E-F N}}, v_{\lambda A_{E-F N}}\right)=\left(1-\left(1-\mu_{A_{E-F N}}\right)^{\lambda},\left(v_{A_{E-F N}}\right)^{\lambda}\right) ; \\
& A_{E-F N}^{\lambda}=\left(\mu_{A_{E-F N}^{\lambda}}, v_{A_{E-F N}^{\lambda}}\right)=\left(\left(\mu_{A_{E-F N}}\right)^{\lambda}, 1-\left(1-v_{A_{E-F N}}\right)^{\lambda}\right), \lambda>0 .
\end{aligned}
$$

Proof of Definition 2 operations are well defined is in Appendix A.2. 
Definition 3. For any E-FNs $A_{E-F N}=\left(\mu_{A_{E-F N}}, v_{A_{E-F N}}\right)$ and $B_{E-F N}=\left(\mu_{B_{E-F N}}, v_{B_{E-F N}}\right)$,

$$
A_{E-F N} \subseteq B_{E-F N} \Longleftrightarrow\left[\mu_{A_{E-F N}} \leq \mu_{B_{E-F N}} \wedge v_{A_{E-F N}} \geq v_{B_{E-F N}}\right] .
$$

An early source of representation of fuzzy subsets can be found in [24,25].

\section{Average and Geometric Operators of E-FNs}

The weighted average operator and the weighted geometric operator of a set of E-FNs are defined. Some of their properties are also outlined.

Definition 4. The weighted average operator for a set of E-FNs is a mapping $E-I F W A$ : $E-F N \times$ $\cdots \times E-F N \rightarrow[0,1]$ given by:

$$
E-I F W A\left(A_{1 E-F N}, \ldots, A_{m E-F N}\right)=\bigoplus_{i=1}^{m} \omega_{i} A_{i E-F N}=\left(1-\prod_{i=1}^{m}\left(1-\mu_{A_{i E-F N}}\right)^{\omega_{i}}, \prod_{i=1}^{m} v_{A_{i E-F N}}^{\omega_{i}}\right)
$$

where $\omega_{i} \geq 0$ for any $1 \leq i \leq m$, and $\sum_{i=1}^{m} \omega_{i}=1$.

Theorem 1. The output of the weighted average operator E-IFWA is an E-FN.

Proof of Theorem 1 is in Appendix A.3.

The above-proposed aggregation operator satisfies the impotency, boundary and monotonicity properties, which are stated below.

Theorem 2. (Idempotency property) The weighted average operator E - IFWA satisfies

$$
E-I F W A\left(A_{1 E-F N}, \ldots, A_{m E-F N}\right)=A_{E-F N}
$$

for a set of equal E-FNs $A_{E-F N}:=A_{1 E-F N}=\cdots=A_{m E-F N}$.

Proof of Theorem 2 is in Appendix A.4.

If we set

$$
\begin{aligned}
& A_{l E-F N}=\left(\mu_{A_{l E-F N}}, v_{A_{l E-F N}}\right)=\left(\min _{1 \leq i \leq m}\left\{\mu_{A_{i E-F N}}\right\}, \max _{1 \leq i \leq m}\left\{v_{A_{i E-F N}}\right\}\right) \\
& A_{u E-F N}=\left(\mu_{A_{u E-F N}}, v_{A_{u E-F N}}\right)=\left(\max _{1 \leq i \leq m}\left\{\mu_{A_{i E-F N}}\right\}, \min _{1 \leq i \leq m}\left\{v_{A_{i E-F N}}\right\}\right),
\end{aligned}
$$

then we have the following result.

Theorem 3. (Boundedness property) The weighted average operator E - IFWA satisfies

$$
A_{l E-F N} \subseteq E-I F W A\left(A_{1 E-F N}, \ldots, A_{m E-F N}\right) \subseteq A_{u E-F N}
$$

for a set of $E$-FNs $A_{1 E-F N}, \ldots, A_{m E-F N}$.

Proof of Theorem 3 is in Appendix A.5.

Theorem 4. (Monotonicity property) Suppose that for any two classes of E-FNS $A_{1 E-F N}, \ldots$, $A_{m E-F N}$ and $B_{1 E-F N}, \ldots, B_{m E-F N}$, it holds that $A_{i E-F N} \subseteq B_{i E-F N}$ for all $1 \leq i \leq m$. Then, the weighted average operator $E-I F W A$ satisfies

$$
E-I F W A\left(A_{1 E-F N}, \ldots, A_{m E-F N}\right) \subseteq E-I F W A\left(B_{1 E-F N}, \ldots, B_{m E-F N}\right) .
$$

Proof of Theorem 4 is in Appendix A.6. 
Definition 5. The weighted geometric operator for a set of E-FNs is a mapping E - IFWA : $E-F N(X) \times \cdots \times E-F N(X) \rightarrow[0,1]$ given by:

$$
E-I F W G\left(A_{1 E-F N}, \ldots, A_{m E-F N}\right)=\bigotimes_{i=1}^{m} A_{i E-F N}^{\omega_{i}}=\left(\prod_{i=1}^{m} \mu_{A_{i E-F N}}^{\omega_{i}} 1-\prod_{i=1}^{m}\left(1-v_{A_{i E-F N}}\right)^{\omega_{i}}\right)
$$

where $\omega_{i} \geq 0$ for any $1 \leq i \leq m$, and $\sum_{i=1}^{m} \omega_{i}=1$.

The weighted geometric operator $E$ - IFWG also satisfies idempotency, monotonicity and boundedness properties.

\section{Score Function of E-FNs}

In what follows, we propose a score function for E-FNs based on the S-norm $\odot_{1}$, and prove its properties.

Definition 6. Given an E-FN $A_{E-F N}$, we define its score function

$$
\begin{aligned}
S c_{\odot_{1}}\left(A_{E-F N}\right) & =\left(1-v_{A_{E-F N}}\right)-\lambda\left(1-\mu_{A_{E-F N}} \odot_{1} v_{A_{E-F N}}\right) \\
& =\left(1-v_{A_{E-F N}}\right)-\lambda\left(1-\left(1-\left(1-\mu_{A_{E-F N}}\right)\left(1-v_{A_{E-F N}}\right)\right)\right),
\end{aligned}
$$

where $\lambda \in[0,1]$.

It is $S c_{\odot_{1}} \in[0,1]$ for any $\mu_{A_{E-F N}}, v_{A_{E-F N}}, \lambda \in[0,1]$. When $\lambda$ increases in $[0,1]$, the score function $S c_{\odot_{1}}$ decreases from the value $1-v_{A_{E-F N}}\left(\right.$ for $\lambda=0$ ) to the value $\mu_{A_{E-F N}}$ $\left(1-v_{A_{E-F N}}\right)($ for $\lambda=1)$.

One of the superiorities of the proposed E-FS score function with respect to the existing p-ROFS score functions, given next in Equations (25)-(31), is that it is defined based on the multiplication of $\mu_{A_{E-F N}}$ and $\left(1-v_{A_{E-F N}}\right),\left(1-v_{A_{E-F N}}\right)\left((1-\lambda)+\lambda \mu_{A_{E-F N}}\right)$, while existing p-ROFS scores are mainly based on the difference $\mu_{A_{E-F N}}-v_{A_{E-F N}}$, which is meaningless in the case $\mu_{A_{E-F N}}=v_{A_{E-F N}}$. In addition, for all $\lambda \in[0,1]$, it can be easily observed from Definition 6 and the S-norm $\odot_{1}$ that

- $\quad S c_{\odot}\left(A_{E-F N}\right)=1$ if $A_{E-F N}=(1,0)$;

- $\quad S c_{\odot}\left(A_{E-F N}\right)=0$ if $A_{E-F N}=(0,1)$.

Thus, the maximum score is obtained for full membership, while complete nonmembership has associated a score value of 0 . We provide below other interesting properties for the proposed score function.

Theorem 5. For any two E-FNs $A_{E-F N}$ and $B_{E-F N}$, if $A_{E-F N} \subseteq B_{E-F N}$, then

$$
S c_{\odot_{1}}\left(A_{E-F N}\right) \leq S c_{\odot_{1}}\left(B_{E-F N}\right) .
$$

Proof of Theorem 5 is in Appendix A.7.

Theorem 6. For any $E-F N A_{E-F N}=\left(\mu_{A_{E-F N}}, v_{A_{E-F N}}\right)$, the score function $S c_{\odot_{1}}\left(A_{E-F N}\right)$ is monotonically increasing with respect to $\mu_{A_{E-F N}}$ and monotonically decreasing with respect to $v_{A_{E-F N}} \cdot$

Proof of Theorem 6 is in Appendix A.8.

Lemma 1. For any $E-F N A_{E-F N}$, the score function $S c_{\odot_{1}}\left(A_{E-F N}\right)$ is a decreasing function of $\lambda$.

Proof of Lemma 1 is in Appendix A.9. 


\section{Decision Making with E-FSs}

Through this section, we first compare the performance of the proposed E-FS score function and the existing p-ROFS scores in the same setting on the pairs of p-ROFS and E-FS datasets. A classical multiple attribute group decision making (MCGDM) problem in which the ranking order of alternatives based on the Evaluation based on Distance from Average Solution (EDAS) method is a worthy topic for this evaluation study.

\subsection{A Critical Analysis of all Existing p-ROFS Score Functions}

In what follows, we first present a set of existing score functions of p-ROFSs, and then compare their outcomes with the proposed E-FS score function from a point of view of finding weaknesses in them. Notice that in all cases discussed the higher the value of a score the more preferable the p-ROFS is.

Let $A_{p-R O F S}=\left\{\left\langle x, \mu_{A_{p-R O F S}}(x), v_{A_{p-R O F S}}(x)\right\rangle: x \in X\right\}$, the following existing p-ROFS score functions in the literature are:

- Yager's [4] score function:

$$
S c_{Y}\left(A_{p-R O F S}\right)=\mu_{A_{p-R O F S}}^{p}(x)-v_{A_{p-R O F S}}^{p}(x)
$$

- Wei et al.'s [13] score function:

$$
S c_{W}\left(A_{p-R O F S}\right)=\frac{1}{2}\left(1+\mu_{A_{p-R O F S}}^{p}(x)-v_{A_{p-R O F S}}^{p}(x)\right) ;
$$

- $\quad$ Peng et al.'s [14] score function:

$$
\begin{aligned}
S c_{P D G}\left(A_{p-R O F S}\right)=\mu_{A_{p-R O F S}}^{p}(x)-v_{A_{p-R O F S}}^{p}(x) & +\left(\frac{e^{\mu_{A_{p-R O F S}}^{p}(x)-v_{A_{p-R O F S}}^{p}(x)}}{e^{\mu_{A_{p-R O F S}}^{p}(x)-v_{A_{p-R O F S}}^{p}(x)}+1}-\frac{1}{2}\right) \\
& \times\left(1-\mu_{A_{p-R O F S}^{p}}^{p}(x)-v_{A_{p-R O F S}}^{p}(x)\right) ;
\end{aligned}
$$

- Mi et al.'s [26] score function:

$$
S c_{M L L}\left(A_{p-R O F S}\right)=\frac{2+\mu_{A_{p-R O F S}}^{p}(x)-v_{A_{p-R O F S}}^{p}(x)}{\left(2-\mu_{A_{p-R O F S}}^{p}(x)+v_{A_{p-R O F S}}^{p}(x)\right) \times\left(2-\mu_{A_{p-R O F S}}^{p}(x)-v_{A_{p-R O F S}}^{p}(x)\right)} ;
$$

- Farhadinia and Liao's [22] score function:

$$
S c_{F L}\left(A_{p-R O F S}\right)=\mu_{A_{p-R O F S}}^{p}(x)+\lambda\left(1-\mu_{A_{p-R O F S}}^{p}(x)-v_{A_{p-R O F S}}^{p}(x)\right), \quad 0 \leq \lambda \leq 1 .
$$

- $\quad$ Peng and Huang's [27] score function:

$$
S c_{P H}\left(A_{p-R O F S}\right)=\mu_{A_{p-R O F S}}^{p}(x)-v_{A_{p-R O F S}}^{p}(x)+\ln \left(2-\mu_{A_{p-R O F S}}^{p}(x)-v_{A_{p-R O F S}}^{p}(x)\right) ;
$$

- $\quad$ Peng and Dai's [14] score function:

$$
\operatorname{Sc}_{P D}\left(A_{p-R O F S}\right)=\frac{\mu_{A_{p-R O F S}}^{p}(x)-2 v_{A_{p-R O F S}}^{p}(x)-1}{3}+\frac{\lambda}{3}\left(\mu_{A_{p-R O F S}}^{p}(x)+v_{A_{p-R O F S}}^{p}(x)+2\right) ;
$$

The above score functions may not provide good performance, even when considering different p-ROFSs. For instance, if we consider p-ROFSs $A=(0.3,0.3)$ and $B=(0.2,0.2)$, then the first, second, third and seventh score functions are not able to distinguish between them when $p=1,2,3$ (refer to Tables 1-3). This is not the case with the proposed new score function (denoted $S c_{F C}$ in Table 4). Furthermore, it is not possible to apply the existing score functions of $\mathrm{p}$-ROFSs to E-FSs that are not $\mathrm{p}$-ROFSs. For instance, if we have $A=(0.9,0.8)$ and $B=(0.2,0.2)$, then the existing score functions when $p=1,2,3$ cannot be applied because, for such $p$-values, $A=(0.9,0.8)$ is not a p-ROFS. Therefore, existing score functions of $\mathrm{p}$-ROFSs are not useful in ranking $A$ and $B$, but we can use the proposed 
new score function (see Table 5). All of these findings indicate that the proposed E-FS score function $S c_{\odot_{1}}$ allows the decision-maker to effectively discount the influence of other score-based decisions.

Table 1. The ranking of $p-\operatorname{ROFS} A=(0.3,0.3)$ and $B=(0.2,0.2)$ with existing score functions with $p=1$.

\begin{tabular}{cccc}
\hline Score Function & $A$-Score & B-Score & Ranking \\
\hline$S c_{Y}$ & 0 & 0 & $A=B$ \\
\hline$S c_{W}$ & 0.5 & 0.5 & $A=B$ \\
\hline$S c_{P D G}$ & 0 & 0 & $A=B$ \\
\hline$S c_{M L L}$ & 0.7143 & 0.625 & $A>B$ \\
\hline$S c_{F L}(\lambda=1)$ & 0.7 & 0.8 & $A<B$ \\
\hline$S c_{P H}$ & -0.3365 & -0.47 & $A>B$ \\
\hline$S c_{P D}$ & 0 & 0 & $A=B$ \\
\hline
\end{tabular}

Table 2. The ranking of $p-\operatorname{ROFS} A=(0.3,0.3)$ and $B=(0.2,0.2)$ with existing score functions with $p=2$.

\begin{tabular}{cccc}
\hline Score Function & $A$-Score & B-Score & Ranking \\
\hline$S c_{Y}$ & & & $A=B$ \\
\hline$S c_{W}$ & 0.5 & 0.5 & $A=B$ \\
\hline$S c_{P D G}$ & 0 & 0 & $A=B$ \\
\hline$S c_{M L L}$ & 0.5495 & 0.5208 & $A>B$ \\
\hline$S c_{F L}(\lambda=1)$ & 0.91 & 0.96 & $A<B$ \\
\hline$S c_{P H}$ & -0.5988 & -0.6523 & $A>B$ \\
\hline$S c_{P D}$ & 0 & 0 & $A=B$ \\
\hline
\end{tabular}

Table 3. The ranking of $p-\operatorname{ROFS} A=(0.3,0.3)$ and $B=(0.2,0.2)$ with existing score functions with $p=3$.

\begin{tabular}{cccc}
\hline Score Function & $A$-Score & B-Score & Ranking \\
\hline$S c_{Y}$ & 0 & 0 & $A=B$ \\
\hline$S c_{W}$ & 0.5 & 0.5 & $A=B$ \\
\hline$S c_{P D G}$ & 0 & 0 & $A=B$ \\
\hline$S c_{M L L}$ & 0.5139 & 0.504 & $A>B$ \\
\hline$S c_{F L}(\lambda=1)$ & 0.973 & 0.992 & $A<B$ \\
\hline$S c_{P H}$ & -0.6658 & -0.6851 & $A>B$ \\
\hline$S c_{P D}$ & 0 & 0 & $A=B$ \\
\hline
\end{tabular}

Table 4. The ranking of $p-$ ROFS $A=(0.3,0.3)$ and $B=(0.2,0.2)$ as extended fuzzy sets (E-FSs) with new score function.

\begin{tabular}{cccc}
\hline$S c_{F C}(\lambda)$ & $A$-Score & B-Score & Ranking \\
\hline$S c_{F C}(\lambda=1)$ & 0.21 & 0.16 & $A>B$ \\
\hline$S c_{F C}(\lambda=1 / 2)$ & 0.455 & 0.48 & $A<B$ \\
\hline$S c_{F C}(\lambda=0)$ & 0.7 & 0.8 & $A<B$ \\
\hline
\end{tabular}


Table 5. The ranking of $A=(0.9,0.8)$ and $B=(0.2,0.2)$ as E-FSs with new score function.

\begin{tabular}{cccc}
\hline$S c_{F C}(\lambda)$ & $A$-Score & B-Score & Ranking \\
\hline$S c_{F C}(\lambda=1)$ & 0.18 & 0.16 & $A>B$ \\
\hline$S c_{F C}(\lambda=1 / 2)$ & 0.19 & 0.48 & $A<B$ \\
\hline$S c_{F C}(\lambda=0)$ & 0.2 & 0.8 & $A<B$ \\
\hline
\end{tabular}

\subsection{E-FS-Based EDAS Technique for MCGDM}

The EDAS model is useful to determine the best alternative(s) corresponding to the biggest value of positive distance from average solution (PDAS) and the smallest value of negative distance from average solution (NDAS), that is, it is useful to deal with conflicting attributes [28].

Suppose that $\left\{R_{1}, R_{2}, \ldots, R_{m}\right\}$ is the set of alternatives, $\left\{C_{1}, C_{2}, \ldots, C_{n}\right\}$ a set of criteria, and $\left\{E_{1}, E_{2}, \ldots, E_{l}\right\}$ a set of experts who evaluate alternatives against criteria using E-FNs

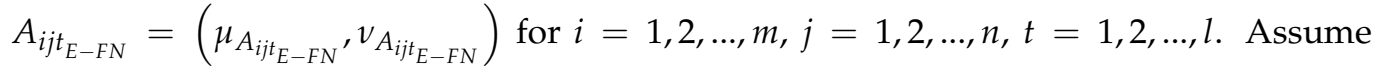
that $\left\{\omega_{E_{1}}, \omega_{E_{2}}, \ldots, \omega_{E_{l}}\right\}$ and $\left\{\omega_{C_{1}}, \omega_{C_{2}}, \ldots, \omega_{C_{n}}\right\}$ are these of weights of experts and criteria, respectively, subject to the constraints: $0 \leq \omega_{E_{t}}, \omega_{C_{j}} \leq 1, \sum_{t=1}^{l} \omega_{E_{t}}=\sum_{j=1}^{n} \omega_{C_{j}}=1$. The E-FS-based EDAS technique for MCGDM problem may be carried out by the following steps:

Step 1. Construct the individual experts' evaluation matrices $A^{t}=\left[A_{i j t_{E-F N}}\right]_{m \times n} t=$ $1,2, \ldots, l$.

Step 2. Apply a weighted aggregation operator, for instance operator $E-I F W A(16)$, to compute the E-FN group matrix $A_{E-F N}=\left[A_{i j_{E-F N}}\right]_{m \times n}$.

Step 3. Compute the following average value for each alternative

$$
\left(\mu_{A_{E-F N}}, v_{A_{E-F N}}\right)_{i}=\left(\sqrt[p]{1-\prod_{j=1}^{n}\left(1-\mu_{A_{E-F N}}^{p}\right)^{\frac{1}{n}}}, \prod_{j=1}^{n}\left(v_{A_{E-F N}}\right)^{\frac{1}{n}}\right), \quad i=1,2, \ldots, m .
$$

Step 4. Apply $S c_{\odot_{1}}$ (23) to derive the positive distance (PD) and negative distance (ND) from $A_{E-F N}$ :

$$
\begin{gathered}
P D\left(\mu_{A_{E-F N}}, v_{A_{E-F N}}\right)_{i j}=\frac{\max \left\{0, S c\left(\left(\mu_{A_{E-F N}}, v_{A_{E-F N}}\right)_{i j}\right)-\mathbf{S c}\left(\left(\mu_{A_{E-F N}}, v_{A_{E-F N}}\right)_{i}\right)\right\}}{\mathbf{S c}\left(\left(\mu_{A_{E-F N}}, v_{A_{E-F N}}\right)_{i}\right)}, \\
N D\left(\mu_{A_{E-F N}}, v_{A_{E-F N}}\right)_{i j}=\frac{\max \left\{0, \mathbf{S c}\left(\left(\mu_{A_{E-F N}}, v_{A_{E-F N}}\right)_{i}\right)-S c\left(\left(\mu_{A_{E-F N}}, v_{A_{E-F N}}\right)_{i j}\right)\right\}}{\operatorname{Sc}\left(\left(\mu_{A_{E-F N}}, v_{A_{E-F N}}\right)_{i}\right)},
\end{gathered}
$$

where $S c$ and Sc stand for the score function of E-FNs and their average E-FN, respectively.

Step 5. Compute the positive weighted distance $P_{i}(i=1,2, \ldots, m)$ and the negative weighted distance $N_{i}(i=1,2, \ldots, m)$ :

$$
\begin{gathered}
P_{i}=\sum_{j=1}^{n} \omega_{C_{j}} P D\left(\mu_{A_{E-F N}}, v_{A_{E-F N}}\right)_{i j^{\prime}} \\
N_{i}=\sum_{j=1}^{n} \omega_{C_{j}} N D\left(\mu_{A_{E-F N}}, v_{A_{E-F N}}\right)_{i j^{\prime}}
\end{gathered}
$$

Step 6. Normalise $P_{i}(i=1,2, \ldots, m)$ and $N_{i}(i=1,2, \ldots, m)$

$$
\bar{P}_{i}=\frac{P_{i}}{\max \left\{P_{1}, P_{2}, \ldots, P_{m}\right\}} ;
$$




$$
\bar{N}_{i}=\frac{N_{i}}{\max \left\{N_{1}, N_{2}, \ldots, N_{m}\right\}} .
$$

Step 7. Compute the integrative appraisal scores

$$
I S c_{i}=\frac{1}{2}\left(\bar{P}_{i}+1-\bar{N}_{i}\right), \quad i=1,2, \ldots, m .
$$

Step 8. Produce the ranking of alternatives, with best alternative being the one with maximum $I S c_{i}$ value.

\subsection{An EDAS-Based Case Study}

A refrigerator is an essential appliance that contributes most in the living standards of a household, although it also consumes a considerable amount of energy due to its continue 24-h running. Therefore, the adequate selection of refrigerator can benefit positively to the individual household by reducing its energy-consumption cost, and to the general environment sustainability on the other side. Below, we consider a decision-making process related to the purchasing of a refrigerator within the context of information dealt with in this paper.

In a recent contribution, Li et al. [29] proposed a MCGDM technique based on the EDAS model with p-ROFS data, which they applied to the application problem of buying an adequate refrigerator based on the following 6 criteria: $C_{1} \equiv$ safety, $C_{2} \equiv$ the performance of refrigeration, $C_{3} \equiv$ designation, $C_{4} \equiv$ the reliability scale, $C_{5} \equiv$ the economical benefit, $C_{6} \equiv$ aesthetics for deciding which of 5 available refrigerators $R_{j}(j=1,2,3,4,5)$ should be bought. All the criteria are of benefit-type, and their corresponding weights are considered as $\omega_{C}=\left(\omega_{C_{1}}, \omega_{C_{2}}, \ldots, \omega_{C_{6}}\right)=(0.20,0.15,0.25,0.17,0.13,0.10)$, and obtained the below $\mathrm{p}$ ROFS collective decision matrix $D$ by applying the q-ROFHA operator, with entry $d_{i j}$ the decision value of the pair $\left(C_{i}, R_{j}\right)$ :

$$
D=\left(\begin{array}{ccccc}
(0.618,0.3734) & (0.5438,0.4149) & (0.3948,0.3148) & (0.5283,0.2186) & (0.546,0.3148) \\
(0.5928,0.4149) & (0.6304,0.362) & (0.4937,0.3148) & (0.5693,0.3037) & (0.3948,0.3148) \\
(0.4937,0.3148) & (0.5928,0.3148) & (0.547,0.1945) & (0.6297,0.3053) & (0.4937,0.4149) \\
(0.3948,0.2133) & (0.6923,0.4149) & (0.5225,0.3148) & (0.3961,0.4334) & (0.4937,0.3148) \\
(0.4937,0.3402) & (0.5925,0.3036) & (0.4651,0.2623) & (0.296,0.4649) & (0.3948,0.3513) \\
(0.4281,0.2572) & (0.6014,0.3169) & (0.5928,0.4149) & (0.3948,0.3148) & (0.4247,0.2856)
\end{array}\right)
$$

With the above p-ROFS collective decision matrix at hand, the average p-ROFS of each of its rows with $p=3$ in (32) results in the following alternatives average p-ROFSs:

$$
\begin{aligned}
& \left(\mu_{A_{p-R O F S}}, v_{A_{p-R O F S}}\right)_{I}=\left(\sqrt[3]{1-\prod_{j=1}^{5}\left(1-\mu_{A_{p-R O F S}}^{3}\right)^{\frac{1}{5}}}, \prod_{j=1}^{5}\left(v_{A_{p-R O F S}}\right)^{\frac{1}{5}}\right), \quad i=1,2, \ldots, 6 \\
& \left(\mu_{A_{p-R O F S}}, v_{A_{p-R O F S}}\right)_{1}=(0.5378,0.32), \quad\left(\mu_{A_{p-R O F S}}, v_{A_{p-R O F S}}\right)_{2}=(0.5514,0.3397), \\
& \left(\mu_{A_{p-R O F S},} v_{A_{p-R O F S}}\right)_{3}=(0.5583,0.3003), \quad\left(\mu_{A_{p-R O F S}}, v_{A_{p-R O F S}}\right)_{4}=(0.5314,0.3281) \text {, } \\
& \left(\mu_{A_{p-R O F S}}, v_{A_{p-R O F S}}\right)_{5}=(0.4725,0.3382), \quad\left(\mu_{A_{p-R O F S}}, v_{A_{p-R O F S}}\right)_{6}=(0.5083,0.3138) \text {. }
\end{aligned}
$$

Table 6 presents the score values of the average p-ROFSs based on the score functions (25)-(31), and the proposed one (23). 
Table 6. Score values of alternatives average p-rung orthopair fuzzy sets (p-ROFSs).

\begin{tabular}{ccccccc}
\hline Score Function & $\left(\mu_{A}, v_{A}\right)_{\mathbf{1}}$ & $\left(\mu_{A}, v_{A}\right)_{\mathbf{2}}$ & $\left(\mu_{A}, v_{A}\right)_{3}$ & $\left(\mu_{A}, v_{A}\right)_{\mathbf{4}}$ & $\left(\mu_{A}, v_{A}\right)_{5}$ & $\left(\mu_{A}, v_{A}\right)_{6}$ \\
\hline $\mathbf{S c} c_{Y}$ & 0.1228 & 0.1284 & 0.1469 & 0.1147 & 0.0668 & 0.1004 \\
$\mathbf{S} \mathbf{c}_{W}$ & 0.5614 & 0.5642 & 0.5735 & 0.5574 & 0.5334 & 0.5502 \\
$\mathbf{S} \mathbf{c}_{M L L}$ & 0.6242 & 0.6342 & 0.6441 & 0.6182 & 0.5761 & 0.6017 \\
$\mathbf{S} \mathbf{c}_{P D G}$ & 0.1477 & 0.1539 & 0.1762 & 0.1381 & 0.0811 & 0.1214 \\
$\mathbf{S} \mathbf{c}_{F L}$ & 0.9672 & 0.9608 & 0.9729 & 0.9647 & 0.9613 & 0.9691 \\
$\mathbf{S} \mathbf{c}_{P H}$ & -0.4715 & -0.4555 & -0.4402 & -0.4811 & -0.5515 & -0.5081 \\
$\mathbf{S} \mathbf{c}_{P D}$ & 0.0614 & 0.0642 & 0.0735 & 0.0574 & 0.0334 & 0.0502 \\
\hline $\mathbf{S c}_{F C}(\lambda=1)$ & 0.3657 & 0.3641 & 0.3906 & 0.3570 & 0.3127 & 0.3488 \\
\hline
\end{tabular}

If we denote the $i j$-th entry of Table 6 by $\left(\mu_{A_{p-R O F S}}, v_{A_{p-R O F S}}\right)_{i j}$, then the score matrices obtained by applying the considered score functions in this contribution are:

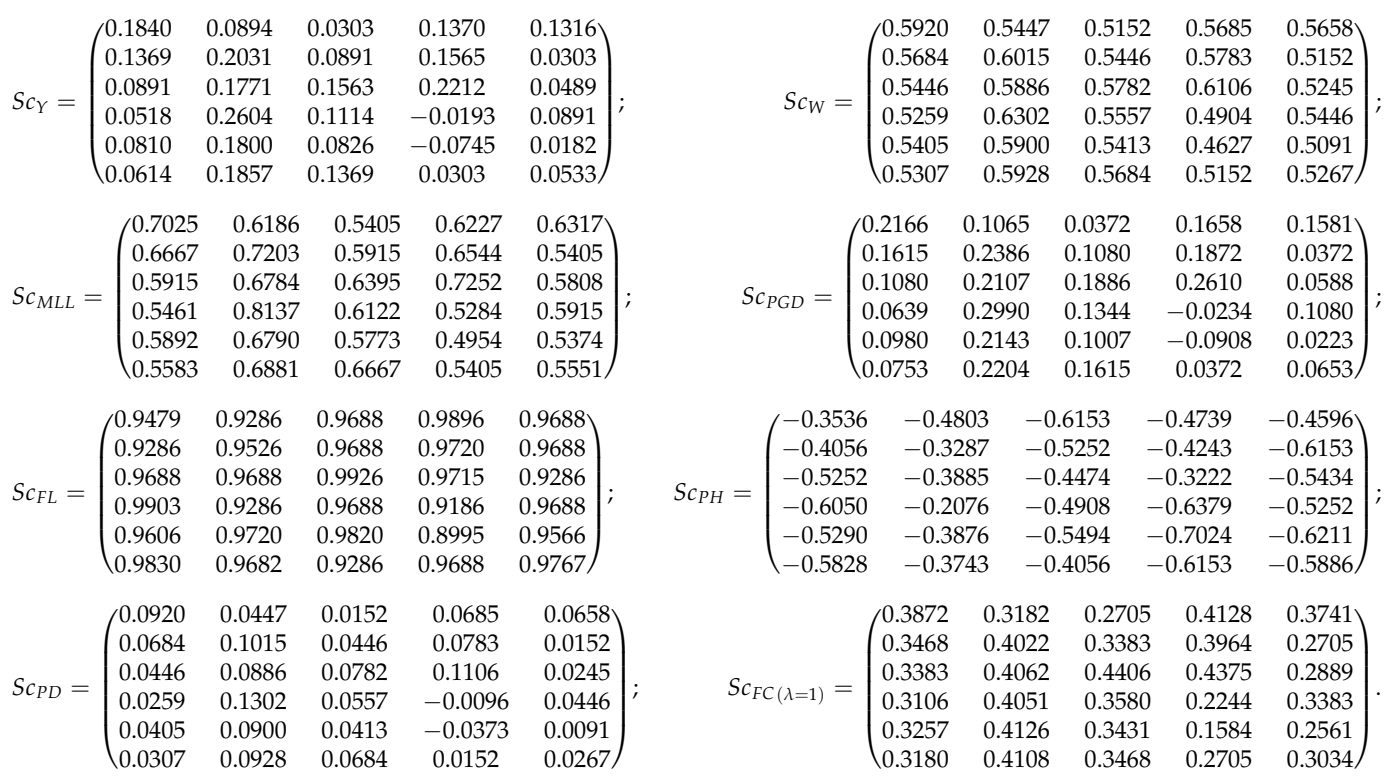

In order to save space, we only provide the PD and ND matrices for $S c_{Y}$ and the proposed $S c_{F C}(\lambda=1)$.

$$
\begin{aligned}
P D S c_{Y}= & \left(\begin{array}{ccccc}
0.4984 & 0 & 0 & 0.1158 & 0.0716 \\
0.0658 & 0.5811 & 0 & 0.2184 & 0 \\
0 & 0.2054 & 0.0638 & 0.5056 & 0 \\
0 & 1.2693 & 0 & 0 & 0 \\
0.2119 & 1.6946 & 0.2359 & 0 & 0 \\
0 & 0.849 & 0.3631 & 0 & 0
\end{array}\right) ; \quad N D S c_{Y}=\left(\begin{array}{ccccc}
0 & 0.2719 & 0.7529 & 0 & 0 \\
0 & 0 & 0.306 & 0 & 0.7638 \\
0.3934 & 0 & 0 & 0 & 0.6671 \\
0.5483 & 0 & 0.0287 & 1.1679 & 0.2231 \\
0 & 0 & 0 & 2.1159 & 0.7278 \\
0.3882 & 0 & 0 & 0.6979 & 0.4692
\end{array}\right) ; \\
P D S c_{F C} & =\left(\begin{array}{cccccccc}
0.0589 & 0 & 0 & 0.1288 & 0.023 \\
0 & 0.1047 & 0 & 0.0888 & 0 \\
0 & 0.0398 & 0.1279 & 0.1198 & 0 \\
0 & 0.1345 & 0.0027 & 0 & 0 \\
0.0417 & 0.3195 & 0.0972 & 0 & 0 \\
0 & 0.1778 & 0 & 0 & 0
\end{array}\right) ; \quad \text { NDS } c_{F C}=\left(\begin{array}{ccccc}
0 & 0.13 & 0.2603 & 0 & 0 \\
0.0474 & 0 & 0.0709 & 0 & 0.257 \\
0.134 & 0 & 0 & 0 & 0.2605 \\
0.1301 & 0 & 0 & 0.3714 & 0.0526 \\
0 & 0 & 0 & 0.4935 & 0.1810 \\
0.0883 & 0 & 0.0056 & 0.2244 & 0.1301
\end{array}\right) .
\end{aligned}
$$

The positive and negative weighted distances, with $\left(\omega_{1}, \omega_{2}, \omega_{3}, \omega_{4}, \omega_{5}, \omega_{6}\right)=$ $(0.2,0.15,0.25,0.17,0.13,0.1)$ adopted from [29], are computed and the corresponding integrative appraisal scores are obtained and reported in Table 7, from which we observe slightly different arrangements of possible refrigerators, based on the existing p-ROFS score functions and the E-FS one, although the best refrigerator is same. Needless to say that if the decision matrix $D$ elements are E-FSs, then existing p-ROFS-based methods would be inapplicable. 
Table 7. Ranking of alternatives with considered score functions.

\begin{tabular}{ccccccc}
\hline $\begin{array}{c}\text { Score-Based EDAS } \\
\text { Method }\end{array}$ & $\boldsymbol{I} \boldsymbol{S} \boldsymbol{c}_{\boldsymbol{1}}$ & $\boldsymbol{I} \boldsymbol{S} \boldsymbol{c}_{\mathbf{2}}$ & $\boldsymbol{I S} \boldsymbol{c}_{\mathbf{3}}$ & $\boldsymbol{I} \boldsymbol{S} \boldsymbol{c}_{\boldsymbol{4}}$ & $\boldsymbol{I} \boldsymbol{S} \boldsymbol{c}_{\mathbf{5}}$ & Ranking Order \\
\hline$S c_{Y}$ (Equation (25)) & 0.392 & 0.95 & 0.3776 & 0.1382 & 0.0868 & $R_{2}>R_{1}>R_{3}>R_{4}>R_{5}$ \\
$S c_{W}$ (Equation (26)) & 0.3489 & 0.9387 & 0.3317 & 0.2326 & 0.013 & $R_{2}>R_{1}>R_{3}>R_{4}>R_{5}$ \\
$S c_{M L L}$ (Equation (28)) & 0.3067 & 0.9874 & 0.2584 & 0.2657 & 0.0096 & $R_{2}>R_{1}>R_{4}>R_{3}>R_{5}$ \\
$S c_{P D G}$ (Equation (27)) & 0.3944 & 0.9488 & 0.3829 & 0.1419 & 0.0902 & $R_{2}>R_{1}>R_{3}>R_{4}>R_{5}$ \\
$S c_{F L}$ (Equation (29)) & 0.4911 & 0.0733 & 0.876 & 0.3131 & 0.2953 & $R_{3}>R_{1}>R_{4}>R_{5}>R_{2}$ \\
$S c_{P H}$ (Equation (30)) & -6.6229 & 23.0165 & -9.6394 & -6.9174 & -20.1849 & $R_{2}>R_{1}>R_{4}>R_{3}>R_{5}$ \\
$S c_{P D}$ (Equation (31)) & 0.392 & 0.95 & 0.3776 & 0.1382 & 0.0868 & $R_{2}>R_{1}>R_{3}>R_{4}>R_{5}$ \\
\hline$S c_{F C}(\lambda=1)$ & 0.3408 & 0.9132 & 0.4978 & 0.3201 & 0.0233 & $R_{2}>R_{3}>R_{1}>R_{4}>R_{5}$ \\
\hline
\end{tabular}

\section{Conclusions}

To deal with the complexity of decision making techniques in practice, this contribution introduced the concept of E-FS, which extends the concept of FS, and the concepts of IFS, PFS together with p-ROFS. The prominent role of E-FS concept is apparent in that the concepts of FS, IFS, PFS and p-ROFS do not make sense in all the situations with uncertainty. Other main contributions of the work are summarised in the following:

1. Development of E-FS algebraic and set operations;

2. Presentation of E-FS average and geometric aggregating operations;

3. Introduction of an E-FS score function.

The direction of the future work of this research may be be focused on different forms of E-FS information aggregation operators and E-FS information measures, decision makers' preference information [30] of E-FSs, individual consensus and group consensus measures [31] of E-FSs.

Author Contributions: Conceptualization, B.F.; methodology, B.F. and F.C.; formal analysis, B.F.; validation, B.F. and F.C.; visualization, B.F and F.C.; writing—original draft preparation, B.F.; writingreview and editing, B.F. and F.C. All authors have read and agreed to the published version of the manuscript.

Funding: This research received no external funding.

Conflicts of Interest: The authors declare that they have no known competing financial interests or personal relationships that could have appeared to influence the work reported in this paper.

\section{Abbreviations}

The following abbreviations are used in this manuscript:

A Classical set (CS)

$A_{F S} \quad$ Fuzzy set (FS)

$A_{\text {IFS }} \quad$ Intuitionistic fuzzy set (IFS)

$A_{P F S} \quad$ Pythagorean fuzzy set (PFS)

$A_{p-R O F S} \quad$ p-rung orthopair fuzzy set (p-ROFS)

$A_{E-F S} \quad$ Extended fuzzy set (E-FS)

$A_{E-F N} \quad$ Extended fuzzy number (E-FN)

\section{Appendix A. Proofs of Main Results}

Appendix A.1. Proof of Proposition 1

Proof. A p-ROFS $A_{p-R O F S}$ is defined as follows:

$$
A_{p-R O F S}=\left\{\left\langle x, \mu_{A_{p-R O F S}}(x), v_{A_{p-R O F S}}(x)\right\rangle: x \in X\right\}
$$


in which the membership degree $\mu_{A_{p-R O F S}} \in[0,1]$ and the non-membership degree $v_{A_{p-R O F S}} \in[0,1]$ satisfy $0 \leq \mu_{A_{p-R O F S}}^{p}(x)+v_{A_{p-R O F S}}^{p}(x) \leq 1$ for any $x \in X$ and $p \in[1, \infty)$. Now, if we set

$$
\left(\mu_{A_{E-F S}}(x), v_{A_{E-F S}}(x)\right)=\left(\mu_{A_{p-R O F S}}^{p}(x), v_{A_{p-R O F S}}^{p}(x)\right),
$$

then, the latter relation implies that:

Case $1\left(\odot:=\odot_{1}\right)$ :

$$
\begin{aligned}
\mu_{A_{E-F S}}(x) \odot_{1} v_{A_{E-F S}}(x) & :=\mu_{A_{E-F S}}(x)+v_{A_{E-F S}}(x)-\mu_{A_{E-F S}}(x) v_{A_{E-F S}}(x) \\
& =1-\left(1-\mu_{A_{E-F S}}(x)\right) \times\left(1-v_{A_{E-F S}}(x)\right) \\
& =1-\left(1-\mu_{A_{p-R O F S}}^{p}(x)\right) \times\left(1-v_{A_{p-R O F S}}^{p}(x)\right) \leq 1 .
\end{aligned}
$$

Case $2\left(\odot:=\odot_{2}\right)$ :

$$
\begin{aligned}
& \mu_{A_{E-F S}}(x) \odot_{2} v_{A_{E-F S}}(x):=\frac{\mu_{A_{E-F S}}(x)+v_{A_{E-F S}}(x)}{1+\mu_{A_{E-F S}}(x) v_{A_{E-F S}}(x)} \\
& =\frac{\mu_{A_{E-F S}}(x)+v_{A_{E-F S}}(x)-\mu_{A_{E-F S}}(x) v_{A_{E-F S} S}(x)+\mu_{A_{E-F S}}(x) v_{A_{E-F S}}(x)}{1+\mu_{A_{E-F S}}(x) v_{A_{E-F S}}(x)} \\
& =\frac{\mu_{A_{p-R O F S}}^{p}(x)+v_{A_{p-R O F S}}^{p}(x)-\mu_{A_{p-R O F S}}^{p}(x) v_{A_{p-R O F S}}^{p}(x)+\mu_{A_{p-R O F S}}^{p}(x) v_{A_{p-R O F S}}^{p}(x)}{1+\mu_{A_{p-R O F S}}^{p}(x) v_{A_{p-R O F S}}^{p}(x)} .
\end{aligned}
$$

Once again, applying (A1), we find that

$$
\mu_{A_{E-F S}}(x) \odot_{2} v_{A_{E-F S}}(x) \leq \frac{1+\mu_{A_{p-R O F S}}^{p}(x) v_{A_{p-R O F S}}^{p}(x)}{1+\mu_{A_{p-R O F S}}^{p}(x) v_{A_{p-R O F S}}^{p}(x)}=1 .
$$

Case $3\left(\odot:=\odot_{3}\right)$ :

$$
\begin{aligned}
& \mu_{A_{E-F S}}(x) \odot_{3} v_{A_{E-F S}}(x):= \\
& \frac{\mu_{A_{E-F S}}(x)+v_{A_{E-F S}}(x)-\mu_{A_{E-F S}}(x) v_{A_{E-F S}}(x)-(1-\epsilon) \mu_{A_{E-F S}}(x) v_{A_{E-F S}}(x)}{1-(1-\epsilon) \mu_{A_{E-F S}}(x) v_{A_{E-F S}}(x)} \\
& =\frac{\mu_{A_{p-R O F S}}^{p}(x)+v_{A_{p-R O F S}}^{p}(x)-\mu_{A_{p-R O F S}}^{p}(x) v_{A_{p-R O F S}}^{p}(x)-(1-\epsilon) \mu_{A_{p-R O F S}}^{p}(x) v_{A_{p-R O F S}}^{p}(x)}{1-(1-\epsilon) \mu_{A_{p-R O F S}}^{p}(x) v_{A_{p-R O F S}}^{p}(x)} .
\end{aligned}
$$

Again, applying equation (A1), we deduce that

$$
\mu_{A_{E-F S}}(x) \odot_{3} v_{A_{E-F S}}(x) \leq \frac{1-(1-\epsilon) \mu_{A_{p-R O F S}}^{p}(x) v_{A_{p-R O F S}}^{p}(x)}{1-(1-\epsilon) \mu_{A_{p-R O F S}}^{p}(x) v_{A_{p-R O F S}}^{p}(x)}=1
$$

for any $\epsilon>0$.

Case $4\left(\odot:=\odot_{4}\right)$ :

$$
\begin{aligned}
\mu_{A_{E-F S}}(x) \odot_{4} v_{A_{E-F S}}(x) \quad:=1-\log _{\epsilon}\left(1+\frac{\left(\epsilon^{1-\mu_{A_{E-F S}}(x)}-1\right)\left(\epsilon^{1-v_{A_{E-F S}}(x)}-1\right)}{\epsilon-1}\right) \\
=1-\log _{\epsilon}\left(1+\frac{\left(\epsilon^{1-\mu_{A_{p-R O F S}}^{p}(x)}-1\right)\left(\epsilon^{1-v_{A_{p-R O F S}^{p}}^{p}(x)}-1\right)}{\epsilon-1}\right) .
\end{aligned}
$$


The fact that $\epsilon>1$ gives rise to $\epsilon-1>0$ together with $\epsilon^{1-\mu_{A_{p-R O F S}}^{p}(x)}-1 \geq 0$ and $\epsilon^{1-v_{A_{p-R O F S}}^{p}(x)}-1 \geq 0$ for any $\mu_{A_{p-R O F S}}^{p}(x), v_{A_{p-R O F S}}^{p}(x) \leq 1$. By taking these outcomes into account, we get $\log _{\epsilon}\left(1+\frac{\left(\epsilon^{1-\mu_{A_{p-R O F S}}^{p}(x)}-1\right)\left(\epsilon^{1-v_{A_{p-R O F S}}^{p}(x)}-1\right)}{\epsilon-1}\right) \geq$ $\log _{\epsilon} 1=0$, and

$$
\mu_{A_{E-F S}}(x) \odot_{4} v_{A_{E-F S}}(x) \leq 1,
$$

for any $\epsilon>1$.

The above results demonstrate that $0 \leq \mu_{A_{p-R O F S}}^{p}(x)+v_{A_{p-R O F S}}^{p}(x) \leq 1$ for any $x \in X$ and $p \in[1, \infty)$ leads to $0 \leq \mu_{A_{E-F S}}(x) \odot v_{A_{E-F S}}(x) \leq 1$, that is, a p-ROFS $A_{p-R O F S}$ is an E-FS $A_{E-F S}$.

\section{Appendix A.2. Proof of Definition 2 Operations Are Well Defined}

Proof. Below, we prove that (8)-(14) are well-defined. It suffices to prove that $0 \leq \mu_{E-F S} \odot_{1} v_{E-F S}$ $\leq 1$ in all cases.

Proof of (8). It is obvious for any E-FN $A_{E-F N}$, we have $0 \leq \mu_{A_{E-F S}} \odot_{1} v_{A_{E-F S}} \leq 1$. Therefore, from the fact that $\mu_{A_{E-F N}^{c}} \odot_{1} v_{A_{E-F N}^{c}}=v_{A_{E-F N}} \odot_{1} \mu_{A_{E-F N}}$, we conclude that $0 \leq$ $\mu_{A_{E-F S}^{c}} \odot_{1} v_{A_{E-F S}^{c}} \leq 1$, that is, $A_{E-F N}^{c}=\left(\mu_{A_{E-F N}^{c}}, v_{A_{E-F N}^{c}}\right)$ is an E-FN.

Proof of (9). Since $A_{E-F N}$ and $B_{E-F N}$ are two E-FNs, that is,

$$
\begin{aligned}
0 \leq \mu_{A_{E-F S}} \odot_{1} v_{A_{E-F S}}:= & \mu_{A_{E-F S}}+v_{A_{E-F S}}-\mu_{A_{E-F S}} v_{A_{E-F S}} \\
& =1-\left[1-\mu_{A_{E-F S}}\right] \times\left[1-v_{A_{E-F S}}\right] \leq 1
\end{aligned}
$$

and

$$
\begin{aligned}
0 \leq \mu_{B_{E-F S}} \odot_{1} v_{B_{E-F S}}:= & \mu_{B_{E-F S}}+v_{B_{E-F S}}-\mu_{B_{E-F S}} v_{B_{E-F S}} \\
& =1-\left[1-\mu_{B_{E-F S}}\right] \times\left[1-v_{B_{E-F S}}\right] \leq 1
\end{aligned}
$$

therefore from definition $A_{E-F N} \cap B_{E-F N}$, it results that

$$
\begin{aligned}
\mu_{A_{E-F N} \cap B_{E-F N}} \odot_{1} v_{A_{E-F N} \cup B_{E-F N}}=\min \left\{\mu_{A_{E-F N}}, \mu_{B_{E-F N}}\right\} \odot_{1} \max \left\{v_{A_{E-F N}}, v_{B_{E-F N}}\right\} \\
=1-\left[1-\min \left\{\mu_{A_{E-F N}}, \mu_{B_{E-F N}}\right\}\right] \times\left[1-\max \left\{\mu_{A_{E-F N}}, \mu_{B_{E-F N}}\right\}\right] \\
=1-\max \left\{1-\mu_{A_{E-F N}}, 1-\mu_{B_{E-F N}}\right\} \times \min \left\{1-\mu_{A_{E-F N}}, 1-\mu_{B_{E-F N}}\right\} .
\end{aligned}
$$

By taking the non-negativity property of all the terms $1-\mu_{A_{E-F N}}, 1-\mu_{B_{E-F N}}, 1-$ $\mu_{A_{E-F N}}$ and $1-\mu_{B_{E-F N}}$ into consideration, we are able to get that

$$
\begin{aligned}
& 0 \leq \mu_{A_{E-F N} \cap B_{E-F N}} \odot{ }_{1} v_{A_{E-F N} \cup B_{E-F N}} \\
& \quad=1-\max \left\{1-\mu_{A_{E-F N}}, 1-\mu_{B_{E-F N}}\right\} \times \min \left\{1-\mu_{A_{E-F N}}, 1-\mu_{B_{E-F N}}\right\} \leq 1 .
\end{aligned}
$$

Proof of (10). The proof is much like that of (9).

Proof of (11). Follows from definition $A_{E-F N} \oplus B_{E-F N}$, we conclude that

$$
\begin{aligned}
& \mu_{A_{E-F N} \oplus B_{E-F N}} \odot_{1} v_{A_{E-F N} \oplus B_{E-F N}}=\left[1-\left(1-\mu_{A_{E-F N}}\right)\left(1-\mu_{B_{E-F N}}\right)\right] \odot_{1}\left[v_{A_{E-F N}} v_{B_{E-F N}}\right] \\
& =1-\left[1-\left[1-\left(1-\mu_{A_{E-F N}}\right)\left(1-\mu_{B_{E-F N}}\right)\right]\right] \times\left[1-\left[v_{A_{E-F N}} v_{B_{E-F N}}\right]\right] \\
& =1-\left(1-\mu_{A_{E-F N}}\right)\left(1-\mu_{B_{E-F N}}\right) \times\left(1-v_{A_{E-F N}} v_{B_{E-F N}}\right) \text {. }
\end{aligned}
$$


Again, the non-negativity property of all the terms $1-\mu_{A_{E-F N}}, 1-\mu_{B_{E-F N}}$ and $1-$ $v_{A_{E-F N}} v_{B_{E-F N}}$ gives rise to

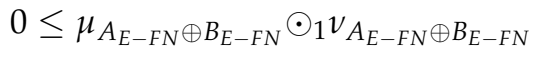

$$
\begin{aligned}
& =1-\left(1-\mu_{A_{E-F N}}\right)\left(1-\mu_{B_{E-F N}}\right) \times\left[1-v_{A_{E-F N}} v_{B_{E-F N}}\right] \leq 1 .
\end{aligned}
$$

Proof of (12). The proof is much like that of (11).

Proof of (13). The proof is immediate from the fact that

$$
\begin{aligned}
0 \leq \mu_{\lambda A_{E-F N}} \odot_{1} v_{\lambda A_{E-F N}} & =1-\left(1-\left[1-\left(1-\mu_{A_{E-F N}}\right)^{\lambda}\right]\right) \times\left[1-\left(v_{A_{E-F N}}\right)^{\lambda}\right] \\
& =1-\left(1-\mu_{A_{E-F N}}\right)^{\lambda} \times\left(1-\left(v_{A_{E-F N}}\right)^{\lambda}\right) \leq 1 .
\end{aligned}
$$

Proof of (14). The proof is much like that of (13).

\section{Appendix A.3. Proof of Theorem 1}

Proof. We prove this by induction on the number of aggregated E-FNs, denoted here by $m$. For $m=2$, from parts of (11) and (13) of Definition 2, we easily conclude that

$$
E-I F W A\left(A_{1 E-F N}, A_{2 E-F N}\right)=\omega_{1} A_{1 E-F N} \oplus \omega_{2} A_{2 E-F N}
$$

is an E-FN, that is,

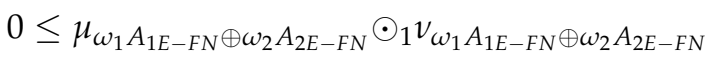

$$
\begin{aligned}
& =\left(1-\left(1-\mu_{A_{1 E-F N}}\right)^{\omega_{1}}\left(1-\mu_{A_{2 E-F N}}\right)^{\omega_{2}}\right) \odot_{1}\left(v_{A_{1 E-F N}}^{\omega_{1}} v_{A_{2 E-F N}}^{\omega_{2}}\right) \\
& =1-\left(1-\left(1-\left(1-\mu_{A_{1 E-F N}}\right)^{\omega_{1}}\left(1-\mu_{A_{2 E-F N}}\right)^{\omega_{2}}\right)\right) \times\left(1-\left(v_{A_{1 E-F N}}^{\omega_{1}} v_{A_{2 E-F N}}^{\omega_{2}}\right)\right) \\
& =1-\left(1-\mu_{A_{1 E-F N}}\right)^{\omega_{1}}\left(1-\mu_{A_{2 E-F N}}\right)^{\omega_{2}} \times\left[1-v_{A_{1 E-F N}}^{\omega_{1}} v_{A_{2 E-F N}}^{\omega_{2}}\right] \leq 1 \text {. }
\end{aligned}
$$

Now, we assume that the result holds for $m$ and prove it for $m+1$. Therefore, we suppose that

$$
E-I F W A\left(A_{1 E-F N}, \ldots, A_{m E-F N}\right)=\bigoplus_{i=1}^{m} \omega_{i} A_{i E-F N}
$$

is an E-FN. We prove now that

$$
E-I F W A\left(A_{1 E-F N}, \ldots, A_{m E-F N}, A_{m+1 E-F N}\right)=\bigoplus_{i=1}^{m+1} \omega_{i} A_{i E-F N}
$$

is also an E-FN. To do this, we have

$$
\begin{aligned}
E-I F W A\left(A_{1 E-F N}, \ldots, A_{m E-F N}, A_{m+1 E-F N}\right) & =\bigoplus_{i=1}^{m+1} \omega_{i} A_{i E-F N} \\
& =\bigoplus_{i=1}^{m} \omega_{i} A_{i E-F N} \oplus \omega_{m+1} A_{m+1 E-F N}
\end{aligned}
$$


in which

$$
\begin{aligned}
0 \leq & \mu_{\oplus_{i=1}^{m}} \omega_{i} A_{i E-F N} \oplus \omega_{m+1} A_{m+1 E-F N} \odot_{1} v_{\oplus_{i=1}^{m}} \omega_{i} A_{i E-F N} \oplus \omega_{m+1} A_{m+1 E-F N} \\
= & \left(1-\prod_{i=1}^{m}\left(1-\mu_{A_{i E-F N}}\right)^{\omega_{i}}\left(1-\mu_{A_{m+1 E-F N}}\right)^{\omega_{m+1}}\right) \odot_{1}\left(\prod_{i=1}^{m} v_{A_{i E-F N}}^{\omega_{i}} \times v_{A_{m+1 E-F N}}^{\omega_{m+1}}\right) \\
= & 1-\left(1-\left(1-\left(1-\prod_{i=1}^{m}\left(1-\mu_{A_{i E-F N}}\right)^{\omega_{i}}\left(1-\mu_{A_{m+1 E-F N}}\right)^{\omega_{m+1}}\right)\right)\right) \\
& \times\left(1-\left(\prod_{i=1}^{m} v_{A_{i E-F N}}^{\omega_{i}} \times v_{A_{m+1 E-F N}}^{\omega_{m+1}}\right)\right) \\
= & 1-\left(1-\prod_{i=1}^{m+1}\left(1-\mu_{A_{i E-F N}}\right)^{\omega_{i}}\right) \times\left(1-\prod_{i=1}^{m+1} v_{A_{i E-F N}}^{\omega_{i}}\right) \leq 1 .
\end{aligned}
$$

This completes the proof.

\section{Appendix A.4. Proof of Theorem 2}

Proof. Taking the equality relationship between all the unified E-FNs, that is, $A_{E-F N}:=$ $A_{1 E-F N}=\cdots=A_{m E-F N}$ together with the condition $\sum_{i=1}^{m} \omega_{i}=1$ in which $\omega_{i} \geq 0$ for any $1 \leq i \leq m$, we conclude that

$$
\begin{aligned}
E-I F W A\left(A_{1 E-F N}, \ldots, A_{m E-F N}\right) & =E-I F W A\left(A_{E-F N}, \ldots, A_{E-F N}\right)=\bigoplus_{i=1}^{m} A_{E-F N}^{\omega_{i}} \\
& =\left(1-\prod_{i=1}^{m}\left(1-\mu_{A_{E-F N}}\right)^{\omega_{i}}, \prod_{i=1}^{m} v_{A_{E-F N}}^{\omega_{i}}\right) \\
& =\left(1-\left(1-\mu_{A_{E-F N}}\right)^{\sum_{i=1}^{m} \omega_{i}}, v_{A_{E-F N} \sum_{i=1}^{m} \omega_{i}}\right) \\
& =\left(1-\left(1-\mu_{A_{E-F N}}\right), v_{A_{E-F N}}\right) \\
& =A_{E-F N} .
\end{aligned}
$$

Appendix A.5. Proof of Theorem 3

Proof. Let $\sum_{i=1}^{m} \omega_{i}=1$ such that $\omega_{i} \geq 0 \forall i$. Denoting

$$
\begin{aligned}
\operatorname{Min}_{\mu}=\min _{1 \leq i \leq m}\left\{\mu_{A_{i E-F N}}\right\} ; & \operatorname{Max}_{\mu}=\max _{1 \leq i \leq m}\left\{\mu_{A_{i E-F N}}\right\} ; \\
\operatorname{Min}_{v}=\min _{1 \leq i \leq m}\left\{v_{A_{i E-F N}}\right\} ; & \operatorname{Max}_{v}=\max _{1 \leq i \leq m}\left\{v_{A_{i E-F N}}\right\} .
\end{aligned}
$$

Since $\omega_{i} \geq 0$, it is:

$$
\begin{gathered}
\left(1-\operatorname{Min}_{\mu}\right)^{\omega_{i}} \geq\left(1-\mu_{A_{i E-F N}}\right)^{\omega_{i}} \geq\left(1-\operatorname{Max}_{\mu}\right)^{\omega_{i}} ; \\
\left(\operatorname{Min}_{v}\right)^{\omega_{i}} \leq\left(v_{A_{i E-F N}}\right)^{\omega_{i}} \leq\left(\operatorname{Max}_{v}\right)^{\omega_{i}},
\end{gathered}
$$

which implies

$$
\begin{gathered}
\left(1-\operatorname{Min}_{\mu}\right)^{\sum_{i=1}^{m} \omega_{i}} \geq \prod_{i=1}^{m}\left(1-\mu_{A_{i E-F N}}\right)^{\omega_{i}} \geq\left(1-\operatorname{Max}_{\mu}\right)^{\sum_{i=1}^{m} \omega_{i}} ; \\
\left(\operatorname{Min}_{v}\right)^{\sum_{i=1}^{m} \omega_{i}} \leq \prod_{i=1}^{m}\left(v_{A_{i E-F N}}\right)^{\omega_{i}} \leq\left(\operatorname{Max}_{v}\right)^{\sum_{i=1}^{m} \omega_{i}} .
\end{gathered}
$$

Since $\sum_{i=1}^{m} \omega_{i}=1$, it is

$$
\begin{gathered}
\operatorname{Min}_{\mu} \leq 1-\prod_{i=1}^{m}\left(1-\mu_{A_{i E-F N}}\right)^{\omega_{i}} \leq \operatorname{Max}_{\mu} \\
\operatorname{Min}_{v} \leq \prod_{i=1}^{m}\left(v_{A_{i E-F N}}\right)^{\omega_{i}} \leq \operatorname{Max}_{v} .
\end{gathered}
$$


From

$$
\operatorname{Min}_{\mu} \leq 1-\prod_{i=1}^{m}\left(1-\mu_{A_{i E-F N}}\right)^{\omega_{i}} \text { and } \prod_{i=1}^{m}\left(v_{A_{i E-F N}}\right)^{\omega_{i}} \leq \operatorname{Max}_{v},
$$

we deduce that

$$
A_{l E-F N} \subseteq E-\operatorname{IFW} A\left(A_{1 E-F N}, \ldots, A_{m E-F N}\right)
$$

while from

$$
\operatorname{Min}_{\mu} \leq 1-\prod_{i=1}^{m}\left(1-\mu_{A_{i E-F N}}\right)^{\omega_{i}} \text { and } \prod_{i=1}^{m}\left(v_{A_{i E-F N}}\right)^{\omega_{i}} \leq \operatorname{Max}_{v},
$$

we deduce that

$$
E-I F W A\left(A_{1 E-F N}, \ldots, A_{m E-F N}\right) \subseteq A_{u E-F N}
$$

which completes the proof.

Appendix A.6. Proof of Theorem 4

Proof. Let $\sum_{i=1}^{m} \omega_{i}=1$ such that $\omega_{i} \geq 0 \forall i$. From Definition 3

$$
A_{E-F N} \subseteq B_{E-F N} \Longleftrightarrow\left[\mu_{A_{E-F N}} \leq \mu_{B_{E-F N}} \wedge v_{A_{E-F N}} \geq v_{B_{E-F N}}\right]
$$

Since $\omega_{i} \geq 0$, it is:

$$
1-\prod_{i=1}^{m}\left(1-\mu_{A_{i E-F N}}\right)^{\omega_{i}} \leq 1-\prod_{i=1}^{m}\left(1-\mu_{B_{i E-F N}}\right)^{\omega_{i}} \wedge \prod_{i=1}^{m}\left(v_{A_{i E-F N}}\right)^{\omega_{i}} \geq \prod_{i=1}^{m}\left(v_{B_{i E-F N}}\right)^{\omega_{i}} .
$$

Therefore, we conclude that

$$
E-I F W A\left(A_{1 E-F N}, \ldots, A_{m E-F N}\right) \subseteq E-I F W A\left(B_{1 E-F N}, \ldots, B_{m E-F N}\right)
$$

Appendix A.7. Proof of Theorem 5

Proof. Since $A_{E-F N} \subseteq B_{E-F N}$, it is

$$
\mu_{A_{E-F N}} \leq \mu_{B_{E-F N}} \wedge v_{A_{E-F N}} \geq v_{B_{E-F N}}
$$

which implies

$$
-\left(1-\mu_{A_{E-F N}}\right) \leq-\left(1-\mu_{B_{E-F N}}\right) \wedge 1-v_{A_{E-F N}} \leq 1-v_{B_{E-F N}} .
$$

Algebraic manipulation lead to the following:

$$
\begin{aligned}
& 1-\left(1-\mu_{A_{E-F N}}\right)\left(1-v_{A_{E-F N}}\right) \leq 1-\left(1-\mu_{B_{E-F N}}\right)\left(1-v_{B_{E-F N}}\right), \\
& 1-\left(\mu_{A_{E-F N}} \odot{ }_{1} v_{A_{E-F N}}\right) \geq 1-\left(\mu_{B_{E-F N}} \odot_{1} v_{B_{E-F N}}\right), \\
& \left(1-v_{A_{E-F N}}\right)-\lambda\left(1-\mu_{A_{E-F N}} \odot_{1} v_{A_{E-F N}}\right) \leq\left(1-v_{B_{E-F N}}\right)-\lambda\left(1-\mu_{B_{E-F N}} \odot_{1} v_{B_{E-F N}}\right) .
\end{aligned}
$$

The latter inequality implies that $S c_{\odot_{1}}\left(A_{E-F N}\right) \leq S c_{\odot_{1}}\left(B_{E-F N}\right)$.

Appendix A.8. Proof of Theorem 6

Proof. The first partial derivatives of

$$
S c_{\odot_{1}}\left(A_{E-F N}\right)=\left(1-v_{A_{E-F N}}\right)-\lambda\left(1-\left(1-\left(1-\mu_{A_{E-F N}}\right)\left(1-v_{A_{E-F N}}\right)\right)\right)
$$


are:

$$
\begin{aligned}
\frac{\partial S c_{\odot_{1}}\left(A_{E-F N}\right)}{\partial \mu_{A_{E-F N}}} & =\lambda\left(1-v_{A_{E-F N}}\right) \\
\frac{\partial S c_{\odot_{1}}\left(A_{E-F N}\right)}{\partial v_{A_{E-F N}}} & =-v_{A_{E-F N}}-\lambda\left(-v_{A_{E-F N}}\right)\left(1-\mu_{A_{E-F N}}\right) \\
& =-v_{A_{E-F N}}\left(1-\lambda\left(1-\mu_{A_{E-F N}}\right)\right) . \\
\text { It is obvious that } \frac{\partial S c_{\odot_{1}}\left(A_{E-F N}\right)}{\partial \mu_{A_{E-F N}}} & \geq 0 \text { while } \frac{\partial S c_{\odot_{1}}\left(A_{E-F N}\right)}{\partial v_{A_{E-F N}}} \leq 0 \text { for any } \lambda \in[0,1] .
\end{aligned}
$$

Appendix A.9. Proof of Lemma 1

Proof. The partial derivatives of $S c_{\odot_{1}}\left(A_{E-F N}\right)$ with respect to $\lambda$ is:

$$
\text { It is obvious that } \frac{\partial S c_{\odot_{1}}\left(A_{E-F N}\right)}{\partial \lambda} \leq 0 \text { for any } \lambda \in[0,1] \text {. }
$$

\section{References}

1. Zadeh, L.A. Fuzzy sets. Inf. Control 1965, 8, 338-353. [CrossRef]

2. Atanassov, K. Intuitionistic Fuzzy Sets, Theory and Applications; Physica-Verlag: Heidelberg, Germany, 1999.

3. Yager, R.R. Pythagorean fuzzy subsets. In Proceedings of the Joint IFSAWorld Congress and NAFIPS Annual Meeting, Edmonton, AB, Canada, 24-28 June 2013; pp. 57-61.

4. Yager, R.R. Generalized orthopair fuzzy sets. IEEE Trans. Fuzzy Syst. 2017, 25, 1222-1230. [CrossRef]

5. Herrera-Viedma, E.; Palomares, I.; Li, C.-C.; Cabrerizo, F.J.; Dong, Y.; Chiclana, F.; Herrera, F. Revisiting Fuzzy and Linguistic Decision-Making: Scenarios and Challenges for Wiser Decisions in a Better Way. IEEE Trans. Syst. Man Cybern. Syst. 2021, 51, 191-208. [CrossRef]

6. Farhadinia B. A novel method of ranking hesitant fuzzy values for multiple attribute decision-making problems. Int. J. Intel. Sys. 2014, 29, 184-205. [CrossRef]

7. Farhadinia, B. Information measures for hesitant fuzzy sets and interval-valued hesitant fuzzy sets. Inf. Sci. 2013, 240, 129-144. [CrossRef]

8. Li, C.-C.; Dong, Y.; Xu, Y.; Chiclana, F.; Herrera-Viedma, E.; Herrera, F. An overview on managing additive consistency of reciprocal preference relations for consistency-driven decision making and Fusion: Taxonomy and future directions. Inf. Fus. 2019, 52, 143-156. [CrossRef]

9. Xu, Z.; Yager, R.R. Some geometric aggregation operators based on intuitionistic fuzzy sets. Int. J. Gen. Syst. 2006, 35, 417-433. [CrossRef]

10. He, Y.; Chen, H.; Zhou, L.; Liu, J.; Tao, Z. Intuitionistic fuzzy geometric interaction averaging operators and their application to multi-criteria decision making. Inf. Sci. 2014, 259, 142-159. [CrossRef]

11. Garg, H. Generalized Pythagorean fuzzy geometric aggregation operators using Einstein t-norm and t-conorm for multicriteria decision-making process. Int. J. Intell. Syst. 2017, 32, 597-630. [CrossRef]

12. Liu, P.; Wang, P. Some p-rung orthopair fuzzy aggregation operators and their applications to multiple-attribute decision making. Int. J. Intell. Syst. 2018, 33, 259-280. [CrossRef]

13. Wei, G.; Gao, H.; Wei, Y. Some p-rung orthopair fuzzy heronian mean operators in multiple attribute decision making. Int. J. Intell. Syst. 2018, 33, 1426-1458. [CrossRef]

14. Peng, X.; Dai, J.; Garg H. Exponential operation and aggregation operator for p-rung orthopair fuzzy set and their decision-making method with a new score function. Int. J. Intell. Syst. 2018, 33, 2255-2282. [CrossRef]

15. Wu, J.; Chiclana, F.; Liao, H. Isomorphic multiplicative transitivity for intuitionistic and interval-valued fuzzy preference relations and its application in deriving their priority vectors. IEEE Trans. Fuzzy Syst. 2018, 26, 193-202. [CrossRef]

16. Garg, H.; Kumar, K. An advanced study on the similarity measures of intuitionistic fuzzy sets based on the set pair analysis theory and their application in decision making. Soft Comput. 2018, 22, 4959-4970. [CrossRef]

17. Wu, J.; Chiclana, F. Multiplicative consistency of intuitionistic reciprocal preference relations and its application to missing values estimation and consensus building. Knowl. Based Sys. 2014, 71, 187-200. [CrossRef]

18. Ureña, R.; Chiclana, F.; Fujita, H.; Herrera-Viedma, E. Confidence-consistency driven group decision making approach with incomplete reciprocal intuitionistic preference relations. Knowl. Based Syst. 2015, 89, 86-96. [CrossRef] 
19. Zhang, X.L. A novel approach based on similarity measure for Pythagorean fuzzy multiple criteria group decision making. Int. J. Intell. Syst. 2016, 31, 593-611. [CrossRef]

20. Tang, G.; Liu, P.; Chiclana, F. A new decision-theoretic rough set model with q-rung orthopair fuzzy information. Appl. Soft Comput. 2020, 91, 106212. [CrossRef]

21. Peng, X. Algorithm for pythagorean fuzzy multi-criteria decision making based on WDBA with new score function. Fundam. Informaticae 2019, 165, 99-137. [CrossRef]

22. Farhadinia, B.; Liao, H. Score-based multiple criteria decision making process by using p-rung orthopair fuzzy sets. Informatica 2020, 1-31. [CrossRef]

23. Farhadinia, B. Study on division and subtraction operations for hesitant fuzzy sets, interval-valued hesitant fuzzy sets and typical dual hesitant fuzzy sets. J. Intell. Fuzzy Syst. 2015, 28, 1393-1402. [CrossRef]

24. Antoni, L.; Krajci, S.; Kridlo, O. Representation of fuzzy subsets by Galois connections. Fuzzy Sets Sys. 2017, 326, 52-68. [CrossRef]

25. Cabrera, I.P.; Cordero, P.; García-Pardo, F.; Ojeda-Aciego, M.; De Baets, B. Galois connections between a fuzzy preordered structure and a general fuzzy structure. IEEE Trans. Fuzzy Syst. 2017, 26, 1274-1287. [CrossRef]

26. Mi, X.; Li, J.; Liao, H.; Kazimieras Zavadskas, E.; Al-Barakati, A.; Barnawi, A.; Taylan, O.; Herrera-Viedma, E. Hospitality brand management by a score-based p-rung orthopair fuzzy V.I.K.O.R. method integrated with the best worst method. Econ.-Res.-Ekon. Istraz. 2019, 32, 3272-3301. [CrossRef]

27. Peng, X.; Huang, H. Fuzzy decision making method based on CoCoSo with critic for financial risk evaluation. Technol. Econ. Dev. Econ. 2020, 2020, 1-30. [CrossRef]

28. Kahraman, C.; Keshavarz, G.M.; Zavadskas, E.K.; Onar, S.C.; Yazdani, M.; Oztaysi, B. Intuitionistic fuzzy EDAS method: An application to solid waste disposal site selection. J. Environ. Eng. Land. Manag. 2017, 25, 1-12. [CrossRef]

29. Li, Z.; Wei, G.; Wang, R.; Wu, J.; Wei, C.; Wei, Y. EDAS method for multiple attribute group decision making under p-rung orthopair fuzzy environment. Technol. Econ. Dev. Econ. 2020, 26, 86-102. [CrossRef]

30. Dong, Y.; Liu, W.; Chiclan, F.; Kou, G.; Herrera-Viedma, E. Are incomplete and self-confident preference relations better? A simulation-based investigation. Inf. Sci. 2019, 492, 40-57. [CrossRef]

31. Liang, H.; Dong, Y.; Ding, Z.; Ureña, R.; Chiclana, F.; Herrera-Viedma, E. Consensus Reaching with Time Constraints and Minimum Adjustments in Group with Bounded Confidence Effects. IEEE Trans. Fuzzy Syst. 2020, 28, 2466-2479. [CrossRef] 